\title{
ENFOQUE BREVE ESTRATÉGICO: UN CAMINO SIMPLE Y EFECTIVO PARA SOLUCIONAR PROBLEMAS COMPLEJOS
}

\section{BRIEF STRATEGIC APPROACH: A SIMPLE AND EFFECTIVE WAY TO SOLVE COMPLEX PROBLEMS}

\author{
Mercedes Castillo Sotelo, Ana Isabel Brito Sánchez y \\ Rubén Vargas Jiménez \\ Universidad Autónoma de Baja California \\ Escuela de Ciencias de la Salud Valle de las Palmas. México
}

Cómo referenciar este artículo/How to reference this article:

Castillo Sotelo, M., Brito Sánchez, A.I. y Vargas Jiménez, R. (2018). Enfoque Breve Estratégico: un camino simple y efectivo para solucionar problemas complejos. Revista de Psicoterapia, 29(110), 257-271.

\section{Resumen}

En el presente informe se presenta el tratamiento y resultados de un proceso terapéutico con una familia constituida por los padres divorciados y dos hijos. El método utilizado fue enfoque Breve Estratégico, cada sesión se utilizó una directiva enfocada al logro de los objetivos, los cuáles eran disminuir la agresión y favorecer interacciones positivas entre los miembros de la familia. Los instrumentos utilizados fueron el genograma, pruebas proyectivas, entrevista individual y familiar. El tratamiento se llevó a cabo en 4 sesiones y se lograron cumplir los objetivos planteados a pesar de que la familia dejó el proceso terapéutico inconcluso. Se concluyó la importancia del uso de directivas como herramientas de la terapia breve así como su efectividad en el logro de cambios y consecución de objetivos a corto plazo.

Palabras clave: Terapia familiar breve estratégica; adolescencia; tareas; familia.

\begin{abstract}
In this study, we present the treatment and results of a therapeutic process with a family that consists of divorced parents and two kids. The method used in this study was Brief Strategic Therapy, and the objectives were to minimize aggression and make for more positive interactions between the family members. The instruments used in this study were the genogram, projective, individual and family clinical interview. The treatment was carried out in 4 sessions and the objectives set were achieved despite the fact that the family left the therapeutic process unfinished. It was concluded in the study, the importance of Solution Focused or Brief Therapy as a tool as well as its effectiveness in achieving change as well as the achievement of short-term goals
\end{abstract}

Keywords: Brief Strategic family therapy; adolescence; directives; family.

Fecha de recepción V1: 01-08-2017. Fecha de recepción V2: 03-11-2017. Fecha de aceptación: 08-04-2018.

Correspondencia sobre este artículo:

E-mail: mercedes.castillo@uabc.edu.mx

Dirección postal: Universidad Autónoma de Baja California. Escuela de Ciencias de la Salud Valle de las PalmaBlvd. Universitario \#1000. Valle de las Palmas, C.P. 21500. Baja California. México

C 2018 Revista de Psicoterapia 


\section{Introducción}

En el presente trabajo se abordará el proceso terapéutico realizado con una familia, la cual acude a consulta debido a que su hijo adolescente es violento física y verbalmente con sus padres y hermano; por otro lado, ha llegado a autoagredirse. Otras características relacionadas con el contexto familiar son: el divorcio de los padres, la discapacidad del hermano menor y la homosexualidad recién verbalizada del adolescente.

El enfoque elegido para trabajar con la familia fue el Familiar Breve Estratégico, debido a que la familia había mostrado poca adherencia a los tratamientos terapéuticos, ellos mencionaron haber acudido a diferentes terapeutas y dejado los procesos inconclusos. En la forma de trabajo que se estableció con esta familia, se optó por el uso de directivas en cada sesión; de esta forma se podría asegurar trabajar con metas a corto plazo, permitiendo que en cada sesión lograran tener un avance. A continuación, se revisará el sustento teórico de la Terapia Breve Estratégica y características asociadas al contexto familiar del caso.

\section{Terapia familiar breve}

Los diferentes enfoques de terapia breve han sido ampliamente desarrollados en los últimos años, éstos buscan sacar a la luz los recursos y soluciones propias con que cuentan las personas. El terapeuta más que enfocarse en patologías y causas de los síntomas, se centra en las habilidades de los clientes que podrán favorecer su propio desarrollo (Haz y Castro, 2011). De acuerdo con Beyebach (2010), pudiera parecer que al decidir utilizar un enfoque de terapias breves está relacionado con aspectos económicos, sin embargo, la elección de un enfoque breve responde más a motivaciones clínicas y éticas del terapeuta.

En Pérez y Rodriguez (2010), Nardone define a la Terapia Breve como el arte de resolver problemas complejos humanos mediante soluciones que parecen simples; es una perspectiva revolucionaria con una teoría compleja y avanzada, que se distingue por su capacidad de producir cambios en tiempos breves. Sostiene que aunque los problemas sean complejos y persistan durante años, no por esto deben requerir soluciones asimismo largas y complicadas. Implica una intervención breve y focalizada, orientada, por un lado, a la extinción de los síntomas y, por el otro, a la reestructuración de la percepción de la persona.

De acuerdo con Hewitt y Gantiva (2009), la terapia breve está encaminada a fomentar la autoeficacia de las personas para lograr la disponibilidad del cambio. En la medida en que una persona incremente su sentido de competencia y eficacia en un contexto específico a través de la terapia, tendrá mayor disponibilidad de cambio y se obtendrán resultados positivos. Cuando las familias empiezan a generar soluciones que funcionan realmente, su confianza y sentido de autoeficacia se afianzan, por lo que podrá tener mayor éxito ante nuevas dificultades. Los enfoques de terapia breve no se limitan a resolver el problema presente, sino que empoderan a la familia o individuos para enfrentarse a diversas situaciones que pueden 
presentarse en el futuro.

\section{Terapia familiar breve estratégica}

La terapia familiar breve estratégica se entiende en torno a tres constructos principales: sistema, patrones de interacción y estrategias. En otras palabras, una familia es un sistema en la que todos sus miembros están interrelacionados y son interdependientes, los miembros de la familia se acostumbran al comportamiento de otros creando patrones de comportamiento. Cuando estos patrones de comportamiento se vuelven rígidos se genera una estructura familiar desadaptativa, la cual tienen un papel importante en la aparición y mantenimiento de problemas de comportamiento, abuso de drogas y otros comportamientos antisociales (Horigian y Szapocznik, 2000).

El objetivo principal que debe de tener un terapeuta estratégico es el de resolver rápidamente los problemas y trastornos que pueden convertirse en invasivos e invalidantes; para lograrlo utiliza las estrategias que le parecen eficaces. De acuerdo con Hewitt y Gantiva (2009) y López (2011), el enfoque de terapia breve se define como una estructura terapéutica, orientada a incrementar la conciencia de los consultantes acerca de sus comportamientos problema, así como de su capacidad y motivación para comenzar a hacer algo al respecto. La meta es ofrecer a los consultantes herramientas para cambiar actitudes básicas y manejar los problemas subyacentes.

En cualquier enfoque terapéutico se busca lograr un cambio en el cliente, por lo que es importante resaltar la definición de cambio dentro de los enfoques breves estratégicos. Nardone (2009) dice que el cambio terapéutico es no casual, sino enfocado en una dirección muy precisa que es: aliviar un sufrimiento, resolver algo que desequilibra al sistema y alcanzar un objetivo prefijado. El cambio no es una iluminación mental (insight), corresponde más bien en un cambio en las percepciones y al cambiar la percepción de la realidad, por ende cambia la reacción ante ella. El objetivo de cualquier terapia con enfoque estratégico es producir cuanto antes un cambio terapéutico: primero se logra el cambio de la percepción; lo cual que modifica la reacción, que a su vez modificará también el comportamiento y la cognición (Pérez y Rodríguez, 2010).

Las problemáticas que presentan las familias, de acuerdo con el enfoque estratégico, están asociadas a las soluciones intentadas para salir del problema, sin embargo, de acuerdo con Cánovas et al. (2014), también existen fuentes generadoras de estrés tanto intrínsecas como extrínsecas que tienen un impacto sobre la familia y su funcionamiento. Éstas no están relacionadas con periodos del ciclo vital, son circunstancias imprevistas, por lo que las familias requieren de un mayor grado de adaptación. Estas situaciones inesperadas ponen en marcha los recursos de las familias para la resolución de conflictos. Cuando después de diversas soluciones intentadas no logran los cambios esperados, acuden en busca de ayuda del profesional. 
Según Colapinto, citado en Vanegas et al. (2012), una familia funcional no se define por la ausencia de estrés, conflicto o problemas, sino por cuán efectivamente los maneja para que no interfieran con su función de promover el bienestar de sus miembros. Una familia "disfuncional" es la que no puede cumplir con esta función. La terapia de este enfoque ayuda a que las familias recuperen la habilidad de manejar las situaciones conflictivas y situaciones estresantes propias del ciclo de la familia. Cuando las familias son capaces de solucionar por si mismos los conflictos, adquieren autoeficacia.

Para lograr este sentimiento de autoeficacia en la familia, en el enfoque breve se utilizan tareas que no sólo se limitan a consolidar los cambios discutidos o producidos en la sesión, sino que deben en lo posible provocar o estimular cambios fuera de ella (Beyebach y Herrero, 2010). De esta forma, los clientes pueden desarrollar este sentido de autoeficacia, pues se dan cuenta que fuera del consultorio, y pueden lograr cambios significativos por sí mismos. Esto resulta ser otro de los factores que contribuye a acortar el tiempo de la terapia.

Por otro lado, las formas específicas de entrevista utilizadas en este enfoque, como las preguntas milagrosas y el escalamiento han resultado ser importantes estrategias para facilitar cambios en los clientes, además de aumentar expectativas y emociones positivas (Franklin, Zhang, Froerer y Johnson, 2017).

La terapia familiar breve estratégica se ha utilizado para ayudar a adolescentes que han presentado diferentes trastornos de conducta, como uso de drogas, bullying, ausentismo escolar, etc. Este enfoque reconoce la importancia de que la familia participe en la mejora del comportamiento y salud mental del niño. Involucrando a la familia en la terapia, se logra ayudar a los padres a generar cambios y reforzar comportamientos, lo cual ha sido un factor de éxito de este enfoque (Killam y Webber, 2013), debido a la utilidad que ha mostrado en el trabajo con adolescentes en su familia, generando cambios de forma rápida que permanecen a largo plazo y permitiendo que las familias adquieran habilidades para enfrentar situaciones presentes y futuras.

\section{Contexto familiar}

De acuerdo con Minuchin (1986), citado Montalvo, Espinosa y Pérez (2013), la familia se desarrolla en el transcurso de cuatro etapas a lo largo de las cuales el sistema familiar sufre variaciones; los períodos de desarrollo pueden provocar transformaciones al sistema y un salto a una etapa nueva y más compleja. Las etapas, reconocidas como el ciclo de vida, son: a) Formación de la pareja. b) La pareja con hijos pequeños. c) La familia con hijos en edad escolar y/o adolescentes. d) La familia con hijos adultos. Cada etapa requiere ajustes del sistema y hay familias que pueden permanecer atoradas en una etapa, llevándolos a la disfuncionalidad familiar y manifestaciones sintomáticas. La familia de estudio se encuentra en diferentes etapas del ciclo vital, por lo que resulta necesario explicar sus características para, posteriormente, contextualizar la intervención. 
En primer lugar, la familia se encuentra con la adolescencia de uno de sus hijos. En esta etapa ocurren una serie de eventos psicológicos en el joven que influyen en sus relaciones familiares y sociales, como son: la búsqueda de autonomía, el estilo personal de relacionarse y la construcción de la identidad. En las familias empiezan disminuir los niveles de cohesión, ya que los jóvenes demandan privacidad e incluso respeto en sus puntos de vista, así sean diferentes a los de la familia en general. Es importante que se lleguen a acuerdos en lugar de a imposiciones por parte de los padres (Vanegas et al., 2012)

La relación comunicativa entre padres e hijos sufre cambios cuando estos se hallan en la adolescencia, lo cual se ve reflejado en la frecuencia de las conversaciones, las cuales, comparadas con las de la infancia, disminuyen. Cabe mencionar que también platican menos de sus relaciones y actividades diarias y conviven poco tiempo. Ante esto, es probable que aparezcan conflictos constantes que antes carecían de importancia. El conflicto, por sí mismo, no es un fenómeno completamente negativo para el desarrollo de la vida familiar, ya que las confrontaciones pueden ayudar a crear un clima propicio para promover la participación e iniciar la reconciliación; sin embargo, unas habilidades ineficientes de negociación pueden provocar el escalamiento del conflicto y llevarlo a niveles disfuncionales (Pérez y Aguilar, 2009).

En la familia de estudio había límites ambivalentes entre el exceso de control y de libertad. Esta situación, de acuerdo con Montolío (2013), puede ser casusa de violencia de hijos a padres, donde los progenitores maltratados advierten que sus recursos educativos no son efectivos. De hecho, cuando utilizan reprimendas o castigos, su hijo responde incrementando, en intensidad y frecuencia, sus conductas violentas. Entonces, la madre y/o padre optan por el camino de la persuasión, la conciliación o negociación que su hijo ignora, incluso, puede reaccionar con mayor desdén porque los considera débiles. En el caso de la familia de estudio el adolescente sabía que su madre cambiaba constantemente las normas y reaccionaba con violencia hacía ella, perdiendo el respeto por la figura materna como autoridad.

De acuerdo con Calvete, Gámez y Orue (2014), la agresión de padres a hijos está relacionado con la cultura de consumo en que son criados los hijos en sociedades occidentales en las que los padres juegan el papel de proveedores. Los hijos buscan la obtención inmediata de sus gratificaciones y la agresión hacia los padres puede representar un medio para alcanzar las metas cuando los padres se niegan a seguir satisfaciendo sus deseos. De acuerdo con estos recientes cambios en los estilos de crianza y desarrollo de los jóvenes, se ha propuesto que la agresión se derivaría de la incapacidad de los padres para establecer límites y consecuencias contingentes a la conducta de sus hijos.

Otra situación a la que esta familia se enfrentó es al divorcio de los padres, quienes llevan separados ya 15 años. De acuerdo con Valdés, Carlos, Urias e Ibarra (2011), citando a diferentes autores, afirman que hay contradicciones sobre el efecto del divorcio en los hijos con motivo de los hallazgos encontrados en diferentes 
estudios. Se encuentran autores que sostienen que el divorcio tiene invariablemente efectos negativos que perduran hasta la vida adulta (Wallerstein, 1998; Sandford, 2006) y existe otro grupo de investigadores que sostienen que no ocasiona efectos negativos, o que cuando los produce desaparecen a mediano plazo (Hetherington, 2003; Shaffer, 2007). Por otro lado, en diversos estudios se ha encontrado que formar parte de una familia de padres separados influye de manera negativa en la asertividad, autoestima, resolución de problemas, intereses vocacionales y en el clima familiar (Rosales y Espinoza, 2012).

La separación de los padres es el resultado de un proceso cuya crisis. Se inicia en la familia a partir de frecuentes contradicciones, que en ocasiones pueden llevar a la violencia intrafamiliar y conyugal, fomentar actitudes y comportamientos no adecuados a nivel psicológico, físico, emocional, cognitivo y social en los miembros que la componen. Estas contradicciones pueden explicar el comportamiento agresivo, la desconfianza, además de la disminuida capacidad para superar dificultades y las pocas destrezas para solucionar conflictos de estos jóvenes que enfrentan la separación de sus padres (Jiménez, Amaris y Valle, 2012).

Valdés, Carlos, Urias e Ibarra (2011) proponen que estas contradicciones descansan en ciertas variables contextuales, que son: la situación socioeconómica después del mismo, el tiempo transcurrido, los conflictos parentales posteriores al divorcio y la participación del padre ausente. En el caso de la familia del estudio, el padre es una figura físicamente periférica que quiere tener el control de la familia, aunado a la discapacidad de su hermano y la mala relación de los padres, ha generado que el divorcio tenga efectos negativos sobre la conducta del adolescente y la dinámica familiar.

Por último, con respecto a las características de la familia, se encuentra la presencia de un miembro con discapacidad. La presencia en la familia de un niño con alguna discapacidad se convierte en un factor potencial que puede perturbar la dinámica familiar (Guevara y González, 2012). Los hermanos también sufren una serie de emociones y cambios de vida, ya que muchas veces recae sobre los ellos mucha responsabilidad hacia el niño con discapacidad; ellos también tienen que sacrificarse, lo que puede provocar problemas de conducta.

La discapacidad del hijo menor es de nacimiento y es más probable que los padres de hijos con discapacidad de nacimiento tengan más desarrollada su capacidad resiliente e indiquen niveles más altos de fortaleza, debido a que han tenido más tiempo de asimilar la discapacidad (Suria, 2013).

En el caso de la familia de estudio, los padres muestran aún dificultad para aceptar la anoftalmia y autismo de su hijo menor y, al no adaptarse a la discapacidad de su hijo, no están generando estrategias que permitan que se desarrolle. Por el contrario, están generando acciones que están estancando su proceso de independencia y autonomía.

A continuación, se revisarán la descripción de la familia, así como de las sesiones que se tuvo con ellos, los instrumentos utilizados y las tareas asignadas. 


\section{Método}

\section{Participantes}

En la tabla 1. se muestran los miembros pertenecientes a la familia de estudio así como ciertas características importantes.

Tabla 1. Miembros de la familia.

\begin{tabular}{lcl}
\hline $\begin{array}{l}\text { Miembro de la } \\
\text { familia }\end{array}$ & Edad & Características \\
\hline Padre & 39 & $\begin{array}{l}\text { Es un hombre de carácter fuerte, se muestra celoso con su } \\
\text { ex esposa y se molesta fácilmente manifestándolo } \\
\text { levantando la voz y moviendo las manos efusivamente }\end{array}$ \\
\hline Madre & 40 & $\begin{array}{l}\text { Tiene una mala relación con su ex esposo, es una mujer } \\
\text { sumisa, dedicada a sus hijos y dependiente económica de } \\
\text { su ex esposo, lo que genera la existencia de una relación } \\
\text { de codependencia y abuso a pesar de estar divorciados } \\
\text { desde hace quince años }\end{array}$ \\
\hline Hijo "Raúl" & 16 & $\begin{array}{l}\text { Es estudiante de preparatoria, cuida muy bien a su } \\
\text { hermano menor fungiendo como hijo parental, sus padres } \\
\text { triangulan con él y esto lo ubica como el chivo expiatorio y } \\
\text { motivo de consulta. }\end{array}$ \\
\hline Hijo menor & 11 & $\begin{array}{l}\text { Tiene 11 años, tiene dos discapacidades; anoftalmia y } \\
\text { autismo, no asiste a la escuela regular y la madre se hace } \\
\text { responsable de todo lo que necesite, generando que sea } \\
\text { dependiente. }\end{array}$ \\
\hline
\end{tabular}

\section{Antecedentes y motivo de consulta}

La familia está desestructurada desde el año 2000, su nivel económico es medio-bajo, con antecedentes de violencia domestica e intentos fallidos de reconciliación de la pareja. El padre es periférico físicamente, pero desea controlar a la familia utilizando su poder económico. La pareja pelea de forma constante y el único punto de acuerdo es que hijo mejor no tiene remedio, por lo que lo hacen dependiente. Raúl (hijo) es la única esperanza, por lo que vierten todas sus expectativas sobre él. Antes de asistir al tratamiento presente, habían recurrido a 7 psicólogos, sin embargo, no habían mostrado constancia con el tratamiento y reportan no haber observado mejorías con ellos.

El motivo de consulta manifiesto es la conducta agresiva que tiene Raúl hacia ellos y el recibir orientación para responder a la homosexualidad de su hijo; es decir que resaltan a Raúl como chivo expiatorio.

\section{Instrumentos}

Se utilizaron diferentes instrumentos para la recogida de información tanto de forma individual, con Raúl (hijo), como a nivel familiar. De forma individual, se 
utilizó entrevista y los instrumentos de evaluación psicológica (ver tabla 2).

Es importante aclarar que las pruebas proyectivas no se apegan al modelo de Terapia Breve Estratégico y, en este caso, no se utilizaron como un medio de diagnóstico si no que se usaron como estrategia para establecer contacto con el adolescente y disminuir la resistencia que mostraba al proceso terapéutico. Al terminar los tests proyectivos, se exploraron con él algunos rasgos representativos de los dibujos y esa situación dio pie a que se pudieran abrir algunos aspectos importantes, uno de ellos fue su homosexualidad y la culpabilidad que sentía por la reacción de su familia. Por otro lado, se habló sobre la agresión reprimida que aparecía como rasgo proyectivo y pudo verbalizarlo. Y el test de la familia se utilizó como catalizador para explorar sobre la dinámica y conflictos familiares.

Tabla 2. Instrumentos utilizados.

\begin{tabular}{ll}
\hline Instrumento & \\
\hline Individuales & Descripción \\
\hline $\begin{array}{l}\text { Test de la } \\
\text { persona bajo } \\
\text { la lluvia }\end{array}$ & $\begin{array}{l}\text { Es una test proyectivo gráfico que resulta útil para evaluar la imagen de } \\
\text { sí mismo que presenta el individuo en condiciones desfavorables. Se } \\
\text { presenta como una situación conflictiva ante la cual el sujeto debe } \\
\text { reaccionar, apelando a los recursos que posee. (Pérez, Scilletta y } \\
\text { Maldavsky, 2013) }\end{array}$ \\
\hline $\begin{array}{l}\text { Test de la } \\
\text { familia }\end{array}$ & $\begin{array}{l}\text { Es un test gráfico que, a través de diferentes indicadores del dibujo se } \\
\text { pueden inferir un conjunto de aspectos sobre la familia, tales como: } \\
\text { tendencias reprimidas, los roles en la familia y los conflictos en el seno } \\
\text { familiar. (Parra, Gomariz, y Sánchez, 2011) }\end{array}$ \\
\hline $\begin{array}{l}\text { Test de } \\
\text { Frases } \\
\text { incompletas }\end{array}$ & $\begin{array}{l}\text { Explora áreas significativas de la adaptación de un individuo a } \\
\text { situaciones especiales o algún conjunto específico de actitudes. } \\
\text { (Quintanar y García, 2013) }\end{array}$ \\
\hline $\begin{array}{l}\text { Test gráfico } \\
\text { de la casa, } \\
\text { árbol y } \\
\text { persona } \\
\text { (HTP) }\end{array}$ & $\begin{array}{l}\text { Se trata de una técnica de evaluación psicológica que aporta un } \\
\text { conocimiento global sobre la personalidad de un individuo (familiar, } \\
\text { afectivo, emocional, autoestima, etc) (Paly, Lolich, Becerra, Azzollini, y } \\
\text { Mara, 2014) }\end{array}$ \\
\hline $\begin{array}{l}\text { Genograma } \\
\text { El genograma permite tener una visión amplia y rápida de patrones } \\
\text { familiares complejos y proporciona una fuente rica de hipótesis acerca } \\
\text { de cómo un problema clínico puede estar conectado con el contexto } \\
\text { familiar del cliente, dando lugar de esta manera a identificar soluciones } \\
\text { intentadas de manera errónea, entre el cliente y los miembros de la } \\
\text { familia. Proporciona además varias direcciones en la cuales se va guiar } \\
\text { al cliente en busca de soluciones (Zamudio, 2015) }\end{array}$ \\
\hline
\end{tabular}

\section{Procedimiento}

A continuación, se hará una descripción de las sesiones incluyendo aquella información que se consideró relevante para el proceso, haciendo énfasis en las tareas que se les dejaron como parte del modelo de Terapia Breve Estratégico. 


\section{Primera sesión}

Objetivos de la sesión:

- Conocer el motivo de consulta y la percepción de los miembros sobre éste.

- Establecer la relación terapéutica.

- Indagar sobre las soluciones previas intentadas para resolver el problema.

En esta primera sesión acudieron puntualmente todos los miembros, se pasó a la pareja Raúl y Magaly, mientras que los hijos se quedaron esperando. Cuando llegaron se preguntó el motivo de consulta, la primera persona que habló fue la madre refiriendo que estaba muy preocupada por la conducta de su hijo Raúl, ya que éste se comporta de forma cada vez más violenta tanto física como verbalmente, se corta a sí mismo en las muñecas de forma superficial y les había confesado su homosexualidad a través de una carta. Por otro lado, el padre se expresa preocupado por la agresividad de Raúl, ya durante el último incidente que habían tenido estuvieron a punto de darse de golpes entre ellos. Ambos coinciden que negarle un permiso es el detonante de la conducta violenta. Lo padres son muy permisivos con su hijo menor y exigentes con Raúl, lo cual justifican con la discapacidad de su hijo menor; por lo cual vuelcan sus expectativas en su hijo mayor. También, manifiestan que, como padres, quieren saber cómo tratar la homosexualidad de su hijo, expresando que lo respetarán, pero con la esperanza de que sea sólo una fase. Comentan que han ido a siete psicólogos, pero deciden dejar de llevarlo porque no obtienen los resultados que esperan.

Posteriormente, se entrevistó a Raúl (hijo) de forma individual, donde manifiesta que lo llevan a terapia porque se porta mal y es grosero, dice que esta situación se da porque quiere salir mucho y no lo dejan. Él minimiza la situación diciendo que lo que pasa no es para tanto como para solicitar ayuda. Con respecto a las cortadas dice que lo hace porque le gusta, pero que su mamá reacciona de forma exagerada. Para el cierre se le preguntó que si estaba de acuerdo en regresar para poder establecer con sus padres nuevas formas de relación y que, así mismo, pudiera obtener más permisos, a lo que respondió que sí. A los padres se les encargó la siguiente tarea.

\section{Tarea:}

"Cinco cualidades" la cual consiste en que los padres hagan una lista de al menos cinco cualidades de sus hijos. Esta tarea se utiliza cuando los padres tienden sólo a concentrarse en aspectos negativos (Beyebach, 2010). Esto generó en los padres un cambio en la percepción que tenían de su hijo, pues tuvieron la oportunidad de rescatar aspectos positivos de él, porque al estar centrados en lo negativo dejaban de verlos. 


\section{Segunda sesión}

Objetivos de la sesión:

- Lograr que Raúl (hijo) acuda a la terapia por voluntad y se involucre activamente en el proceso terapéutico.

- Conocer la percepción de Raúl sobre la situación familiar.

- Indagar las situaciones en que se dan las conductas autolesivas.

Como estrategia para logar que Raúl (hijo) se involucre activamente en el proceso terapéutico, se decidió tener una sesión individual para conocer la perspectiva que él tenía de la situación. Se indagó sobre su conducta para encontrar el detonante de su autoagresión. Refiere que se enoja mucho cuando no le dan permiso para salir, se siente impotente porque no encuentra una razón convincente para que se lo nieguen, la frustración ante esta situación lo lleva a cortarse las muñecas de forma superficial.

La única justificación que le da su papá es que le sale muy caro mantenerlo como para darle dinero para salir y que además no lo va a ir a recoger. Cuando lo llegan a dejar es la mamá quien va por él. Comenta que su papá siempre está ocupado y sin dinero. La mamá se pasa cuidando a su hermano y cuando ella sale, su papá asume que está saliendo con otra persona. Cuando esto sucede su papá trata de obtener información de él, por lo que se siente involucrado en la relación de pareja. Raúl comenta que no tiene su propio espacio por lo que se siente molesto, pues su madre lo obliga a ir a todas las actividades de su hermano.

Al hablar de las autolesiones, se le preguntó cómo había reaccionado su mamá y dijo que enojada, se le cuestionó sobre si era la reacción que esperaba y comentó que no, que él buscaba la atención y preocupación de su madre, mas no generarse algún daño. Con eso, se descartó el temor de la madre a que su hijo se suicide.

\section{Tarea:}

La carta a los padres: Consiste en pedirle al adolescente que cuando se sienta lastimado o tratado injustamente por sus padres escriba una carta expresando todas sus emociones negativas. Después de escribirla, hará una segunda ya más calmada y matizada, explicando a sus padres qué es lo que le ha molestado, les pedirá un cambio concreto que espera de ellos y, a su vez, se comprometerá a hacer algo diferente. La primera carta se destruye y la segunda puede entregarse a los padres (Beyebach, 2010). Esta tarea le permitió a Raúl dentro de la sesión darse cuenta de qué es lo que le molestó de la conducta de su madre y poder identificar un cambio concreto de conducta que espera en ella así mismo, se concientizó que para lograr un cambio en su madre debía lograr un cambio en él. De otra forma, la tarea se quedó como una herramienta para controlar su agresión hacia sus padres e identificar y expresar lo que espera de ellos de forma más tranquila y asertiva. 


\section{Tercera sesión}

Objetivos de la sesión:

- Utilizar las pruebas proyectivas para establecer contacto con él y disminuir sus resistencias.

- Rescatar la figura del terapeuta.

- Conocer el motivo de consulta para Raúl.

En esta sesión se le aplicaron algunas pruebas proyectivas a Raúl. Dentro de la sesión comenta que ya no quiere ir, que está harto de que su familia sólo lo lleve a él. Eso abrió la pauta para que se indagara sobre sus experiencias anteriores en terapia, se le preguntó que si alguno de sus otros psicólogos lo había ayudado en algo, a lo que contestó que no. Después, se le confrontó con que si estaba seguro sobre si alguno de ellos le había ayudado a reconocer su homosexualidad y aceptarla (primera vez que se tocó el tema con él), a lo que contestó que sí, que el último. Comentó que también le ayudó a aceptar que necesitaba ser menos cerrado socialmente, se dio cuenta que tenía dificultades para socializar, a pesar de que él quiere tener muchos amigos.

En esta sesión se puede ver como el mismo Raúl abre el motivo de consulta, mostrando resistencia a continuar en el rol que se le ha asignado dentro de su familia. Por otro lado, al encontrar que sí había recibido ayuda, se pudo rescatar la figura del terapeuta.

\section{Tarea:}

"La tarea de fórmula de primera sesión" que consistió en darle a Raúl la siguiente prescripción: "Me gustaría que observaras en tu familia aquellas cosas que quieres que sigan ocurriendo". Esta tarea se utiliza cuando en la entrevista y primeras sesiones no se ha logrado obtener información útil sobre las excepciones y avances. En esta familia en que todos los miembros tienden a ver los aspectos negativos les permitirá ver aspectos positivos, recursos y fortalezas de su familia.

\section{Cuarta sesión}

Objetivos de la sesión:

- Identificar los avances y mejorías logradas hasta el momento.

- Establecer mejores patrones de comunicación entre Raúl y su madre.

- Establecer compromisos entre sus miembros para mejorar las relaciones familiares.

En esta sesión llegan sólo Magaly (mamá) Raúl (hijo) y Jesús (hijo menor). La mamá explicó que se había peleado una noche antes con Raúl (papá) y por eso no asistiría. Cuando venían de camino, el papá agredió a Raúl y le dijo que, por culpa de su mamá, él no iba a poder estar en la sesión de hoy, pero que lo apoyaba y lo quería mucho. 
Se llevó a cabo con ellos una recapitulación de las sesiones anteriores, recordando el motivo de consulta inicial, en ese momento la mamá comenta que Raúl ha mejorado mucho, que ya no es tan contestón. Se le preguntó en qué otros aspectos había mejorado su hijo, dijo que en todos, menos en que se seguía cortando y eso la tenía preocupada todavía. En ese momento, se aprovechó para preguntarle cómo había reaccionado cuando se dio cuenta que se seguía cortando, a lo que contestó que enojada y que, por eso, decidió pegarle y castigarlo. En ese momento, Raúl le contestó que no le había gustado que se enojara, que él quería que se sintiera preocupada y no enojada. A lo que madre le contestó que estaba enojada porque ella estaba haciendo todo por darle una buena vida y que, al hacer eso, le estaba haciendo sentir como si estuviera fallando.

Para mejorar la situación Raúl se comprometió a hacer los quehaceres que su mamá le pidiera, no sería contestón, se trataría de controlar con los corajes y que obedecería. Por su lado, la madre dijo que estaba dispuesta a darle permisos y una vez que se los haya dado no amenazarlo constantemente con quitárselos.

\section{Tarea:}

"Un milagro por la mañana" Se les dijo dentro de la sesión a los miembros presentes que, al levantarse, mientras se están bañando y arreglando, se imaginen que sucede un milagro y que todos los problemas que los traen a terapia se resuelven. ¿Qué sería distinto?, ¿qué harían diferente? Luego se les pidió que entre todo lo que han pensado escojan una cosa, la más pequeña e insignificante de todas ellas y la lleven a cabo, fijándose en el efecto que produce (Beyebach, 2010). Esta tarea busca llevar a la acción a los clientes, introducen expectativas de cambio lo que a su vez lo genera.

\section{Resultados}

A pesar de que se vio a la familia durante sólo cuatro sesiones y ellos mismos fueron quienes tomaron la decisión de dejar de ir a la terapia, se lograron resultados específicos. Después de la última sesión que se tuvo con la familia, se tuvieron contactos telefónicos posteriores que hacía la familia para cancelar las citas, primero comentando que todo estaba bien y sin problemas, después refirieron sobre algunos cambios que se habían generado dentro de la dinámica familia. El más importante para la familia fue la relación madre e hijo, dónde la mamá y Raúl lograron llegar a acuerdos sobre los permisos sin que la mamá trate de controlarlo a través de ellos y sin que Raúl reaccione agresivo ante ella.

También se pudo observar una mejoría en la relación padre-hijo, porque en la última sesión, a pesar de que el papá no acudió, le llamó y le expresó su amor y apoyo directamente, sin utilizar a su mamá como intermediaria.

Las conductas agresivas de Raúl disminuyeron, la comunicación con su madre mejoró y ella pudo establecer límites más claros, recuperando la autoridad frente a su hijo. 
Se hizo un seguimiento telefónico a los seis meses para constatar la permanencia de los cambios que habían logrado como familia. El contacto fue con la madre, quien refiere que la relación con su hijo mejoró mucho, que aprendieron a negociar y a dialogar para resolver los conflictos. Comenta que en ocasiones se siguen enojando, pero logran llegar a acuerdos y resolverlos. Por otro lado, expresa estar muy contenta debido a que su hijo dejó de cortarse a sí mismo. Con respecto al hijo menor las cosas parecen seguir igual, la madre lo sigue sobreprotegiendo, pero logrando un equilibrio en darle atención a sus dos hijos. Al momento del último seguimiento, no se encontraban acudiendo a otro proceso terapéutico, sólo mencionaron que de ser necesario volverían a iniciar su proceso con el mismo terapeuta.

\section{Discusión}

Si bien existen otras situaciones conflictivas dentro del ambiente familiar con las tareas y prescripciones indicadas dentro de las sesiones, se logró generar cambios en la dinámica e interacción familiar, se empoderó a la familia y se generó el sentido de autoeficacia que les podrá ayudar a resolver conflictos posteriores. Esta es una de las principales metas de la terapia con enfoque breve estratégico, por lo que, a pesar de que fueron pocas sesiones, se lograron los objetivos del enfoque y, a su vez, se logró resolver la situación conflictiva que aquejaba a los miembros de la familia.

Se cree que los cambios terapéuticos logrados fueron en su mayoría debido a las tareas y prescripciones utilizadas, ya que éstas estaban enfocadas en que la familia reconociera sus recursos y los utilizara en su beneficio. Por otro lado las tareas tenían como objetivo mejorar las interacciones entre sus miembros y mejorar la comunicación. Ésta última, es de acuerdo a las investigaciones previas una de las mayores dificultades a las que se enfrentan las familias con hijos adolescentes, por lo que abrir los canales de comunicación y la capacidad de negociación es de las tareas más importantes que deben lograr las familias en esta etapa.

Con este caso, se puede rescatar que los problemas complejos que aquejan a las familias no requieren necesariamente intervenciones complicadas, ni largas. Con intervenciones simples, pero enfocadas a las necesidades específicas de la familia, y con los objetivos claros de lo que se busca lograr con las mismas, se pueden generar cambios duraderos en las familias e individuos.

Por otro lado, se recomienda que durante las sesiones no se deje de lado a algún miembro de la familia, como en este caso se hizo con el hermano menor; siendo un aspecto importante que se debía trabajar. Pues tal como se mencionan en estudios realizados, la discapacidad de un hijo genera dificultades a los hermanos. Entonces, ayudar a la familia a favorecer el desarrollo de su hijo menor podría también continuar favoreciendo los cambios con su hijo mayor. 


\section{Referencias bibliográficas}

Beyebach, M. (2010). 24 ideas para una psicoterapia breve. España: Herder.

Beyebach, M y Herrero, M. (2010). 200 tareas en terapia breve. España: Herder.

Calvete, E., Gámez, M. y Orue, I. (2014). Características familiares asociadas a violencia filio parental en adolescentes. Anales de psicología, 30(3), 1176-1182. Recuperado de: https://bit.ly/2tyCDDx

Cánovas, P., Sahuquillo, M., Císcar, E. y Martínez, C. (2014). Estrategias de intervención socioeducativa con familias: Análisis de la orientación familiar en los servicios especializados de atención a la familia e infancia de la comunidad valenciana. Educación XX1, 17(2), 265-288. doi: 10.5944/educxx1.17.2.1149

Franklin, C., Zhang, A., Froerer, A. y Johnson, S. (2017). Solution Focused brief therapy: a systematic review and meta-summary of process research. Journal of Marital and Family Therapy, 43(1), 16-30. Recuperado de: http://onlinelibrary.wiley.com/doi/10.1111/jmft.12193/full

Guevara, Y. y González, S. (2012). Las familias ante la discapacidad. Revista Electrónica de Psicología Iztacala, 15(3), 10-23. Recuperado de: http://www.medigraphic.com/

Haz, A. y Castro, S. (2011). Enfoques de soluciones. En búsqueda de los recursos y habilidades delos clientes. Psykhe, 3(1). Recuperado de: http://www.psykhe.cl/

Hewitt, N. y Gantiva, A. (2009). La terapia breve: una alternativa de intervención psicológica efectiva. Avances en Psicología Latinoamericana, 27(1), 165-176. Recuperado de: http://www.scielo.org.co/

Horigian, V. E. y Szapocznik, J. (2015). Brief strategic family therapy: Thirty-five years of interplay among theory, research, and practice in adolescent behavior problems. Clinical Child and Family Psycholy Review, 3(2), 117-134. Recuperado de: https://www.ncbi.nlm.nih.gov/pmc/articles/PMC1480650/

Jiménez, M., Amaris, M. y Valle, M. (2012). Afrontamiento en crisis familiares. Salud Uninorte, 28(1), 99-112. Recuperado de: http://www.scielo.org.co

Killam, W. K. y Weber, B. (2013). The Effectiveness of Brief Strategic Family Therapy With At-Risk African American Adolescents. VISTAS, 1-10. Disponible en: https://bit.ly/2lyJuII

López, P. (2011). Intervención estratégica con familias: reducir la complejidad para potenciar los recursos. $E d u$ cación social: revista de intervención socioeducativa, 49, 48-69. Recuperado de: https://dialnet.unirioja.es/

Montalvo, J. Espinosa, M. y Pérez, A. (2013). Análisis del ciclo vital de la estructura familiar y sus principales problemas en algunas familias mexicanas. Alternativas en Psicología, 17(28), 73-91. Recuperado de: http://pepsic.bvsalud.org/

Montolío, C. (2013). La violencia de hijos adolescentes contra sus progenitores. Revista sobre la infancia y la adolescencia, 5, 12-30. doi: http://dx.doi.org/10.4995/reinad.2013.1571

Nardone, G. y Balbi, E. (2009). Surcar el mar sin que el cielo lo sepa. Lecciones sobre el cambio terapéutico y las lógicas no ordinarias. Barcelona, España: Herder.

Paly, G., Lolich, M., Becerra, L., Azzollini, S. y Mara, N. (2014). Indicadores de personalidad del HTP en veteranos y ex-combatientes de la Guerra de Malvinas. Psicología Clínica y Psicoterapia: Psicologíacientífica.com, 17(5). Recuperado de: http://www.psicologiacientifica.com/

Parra, J., Gomariz, M. y Sánchez, M. (2011). El análisis del contexto familiar en la educación. Revista electrónica interuniversitaria de formación del profesorado,14(1), 177-192. Recuperado de: https://bit.ly/2KjYkAA

Pérez, M. y Aguilar, J. (2009). Relaciones del conflicto padres-adolescentes con la flexibilidad familiar, comunicación y satisfacción marital. Psicología y salud, 19(1), 111-120. Recuperado de: https://bit.ly/2tBxDgy

Pérez, C. y Rodríguez, P. (2010). Entrevista a Giorgio Nardone Terapia Breve Estratégica: Una Realidad Inventada que Produce Efectos. Clínica Contemporánea, 1(1), 61-66. Recuperado de: http://www.copmadrid.org

Rosales, C. y Espinoza, M. (2012). La percepción del clima familiar en adolescentes miembros de diferentes tipos de familias. Psicología y Ciencia Social/Psychology and Social Science, 10(1), 64-71. Recuperado de: http://tuxchi.iztacala.unam.mx/

Suria, R. (2013). Análisis comparativo de la fortaleza en padres de hijos con discapacidad en función de la tipología y la etapa en la que se adquiere la discapacidad. Anuario de psicología/The UB Journal of psychology, 43(1), 23-37. Recuperado de: http://www.raco.cat/index.php/AnuarioPsicologia/article/view/264842

Suria, R. (2014). Análisis del empoderamiento en padres y madres de hijos con discapacidad. Boletín de psicología, 110, 83-98. Recuperado de: https://dialnet.unirioja.es/servlet/articulo?codigo $=4638971$

Valdés, A., Carlos, E., Urias, M. e Ibarra, B. (2011). Efectos del divorcio de lospadres en el desempeño académico y la conducta de los hijos. Enseñanza e Investigación en Psicología, 16(2), 295-308. Recuperado de: https://dialnet.unirioja.es/servlet/articulo? codigo $=4638971$ 
Vanegas, G., Barbosa, A., Alfonso, M., Delgado, L. y Gutiérrez, J. (2012). Familias monoparentales con hijos adolescentes y psicoterapia sistémica: Una experiencia de intervención e investigación. Revista Vanguardia Psicológica Clínica Teórica y Práctica, 2(2), 203-215. Recuperado de: https://bit.ly/2Ip3C8Y

Pérez, S., Scilletta, D. y Maldavsky, D. (2013). Análisis de la respuesta verbal al test de persona bajo la lluvia. Aplicación del Algoritmo David Liberman (ADL). Subjetividady procesos cognitivos, 17(1), 206-231.

Quintanar, F. y García, C. (2013). Perfil de representaciones de la vejez mediante un test proyectivo de frases incompletas (FIIRAV-I). Psicología y salud, 23(1), 33-43.

Zamudio, S. (2015). Terapia centrada en soluciones aplicada a la agresión sexual. Estudio de caso. Ajayu Órgano de Difusión Cientifica del Departamento de Psicología UCBSP, 9(1), 132-168. Recuperado de: http:// www.scielo.org.bo/ 